\title{
In vivo Tissue Engineering: Mimicry of Homing Factors for Self-Endothelialization of Blood-Contacting Materials
}

\author{
Meltem Avci-Adali Heidi Stoll Nadja Wilhelm Nadja Perle \\ Christian Schlensak Hans P. Wendel \\ Clinical Research Laboratory, Department of Thoracic, Cardiac and Vascular Surgery, University Hospital Tuebingen, \\ Tuebingen, Germany
}

\section{Key Words}

Blood-contacting materials - Endothelial progenitor cells . Homing factor mimicry

\begin{abstract}
Thrombogenicity of foreign surfaces is the major obstacle in cardiovascular interventions. Despite enormous advances in biomaterials research, the hemocompatibility of blood-contacting materials is still not satisfactory and the native endothelium still represents the ideal surface for blood contact. Circulating adult endothelial progenitor cells (EPCs) in the human blood provide an excellent source of autologous stem cells for the in vivo self-endothelialization of bloodcontacting materials. For this purpose, material surfaces can be coated with capture molecules mimicking natural homing factors to attract circulating EPCs. Hitherto, several ligands, such as aptamers, monoclonal antibodies, peptides, selectins and their ligands, or magnetic molecules, are used to biofunctionalize surfaces for the capturing of EPCs directly from patient's bloodstream onto blood-contacting materials. Subsequently, attracted EPCs can differentiate into endothelial cells and generate an autologous endothelium. The in vivo self-endothelialization of blood-contacting materials prevents the recognition of them as a foreign body; this opens up revolutionary new prospects for future clinical stem-cell and tissue engineering strategies.
\end{abstract}

Copyright $\odot 2013$ S. Karger AG, Basel

\section{KARGER}

E-Mail karger@karger.com

www.karger.com/pat

\section{Introduction}

Clinically applied blood-contacting intra- or extracorporeal devices, implants or grafts are mainly made of metals, alloys or synthetic polymers such as expandedpolytetrafluoroethylene (e-PTFE, Gore-Tex ${ }^{\circledR}$ ) or polyethylene terephthalate (PET, Dacron ${ }^{\circledR}$ ). These materials were originally used for industrial applications and, due to their excellent mechanical and physical properties, later found their way into clinical surgery. However, the hemocompatibility of these materials for biomedical applications is still not satisfactory; to improve it, artificial surfaces need several optimizations, such as biocompatible surface coatings and modifications [1]. Hitherto, material surfaces were coated with anticoagulants, such as heparin, hirudin, thrombomodulin or tissue-factor pathway inhibitor (TFPI) or with other molecules, such as nitrogen monoxide donors, polyethylene glycol (PEG), glycosaminoglycanes, phosphorylcholine or albumin, to prevent the adhesion and activation of thrombocytes [2].

In spite of several improvements, the native endothelium still represents the ideal surface for blood contact. For this purpose, implants can be colonized in vitro with autologous endothelial cells obtained from a vein biopsy of a patient. However, this method is very labor-intensive, expensive and carries the risk of bacterial contamination and cell alteration.

Prof. Hans Peter Wendel, $\mathrm{PhD}$

Clinical Research Laboratory, Department of Thoracic, Cardiac and Vascular Surgery, University Hospital Tuebingen, Calwerstrasse 7/1 DE-72076 Tuebingen (Germany)

E-Mail hans-peter.wendel@med.uni-tuebingen.de 
Fortunately, adults still have reserves of stem cells in their body for regenerating cells and repairing injured or damaged tissues. Of these stem cells, the endothelial progenitor cells (EPCs) have the capability to differentiate into endothelial cells, to repair endothelial injuries and contribute to angiogenesis and neovasculogenesis. Thus, EPCs represent a unique source of stem cells for the endothelialization of implants.

Nowadays, simple and ready-to-use implants or devices are required in clinical surgery to achieve rapid endothelialization, good reproducibility and treatment success. One promising way to achieve this is the use of biofunctionalized implants that are coated with EPC-specific ligands, called capture molecules. Examples of such ligands are aptamers, monoclonal antibodies (MABs), peptides, selectins and their ligands or magnetic molecules (fig. 1). These biofunctionalized ready-to-use implants containing EPC homing-factor-mimetic capture molecules can then be implanted into the patients. These molecules can attract circulating EPCs directly from the patient's blood and lead to the in vivo colonization of implants with stem cells. Captured EPCs can differentiate into endothelial cells and can generate an optimal physiological surface for the patient, namely an autologous endothelium.

However, to ensure the successful capturing of EPCs from the circulating blood, additional surface coatings are necessary to prevent unspecific adsorption of serum proteins and adhesion of thrombocytes, which can cover the capture molecules within a very short time and make them inaccessible to EPCs. Furthermore, the material surfaces should support the adhesion and spreading of the EPCs.

\section{Different Capturing Molecules for Biofunctionalization of Blood-Contacting Surfaces}

\section{EPC-Specific Aptamers}

Aptamers are short, single-stranded RNA or DNA oligonucleotides with a length of up to 100 nucleotides. Due to their 3-dimensional structure, they are able to bind their target with high affinity and specificity. Aptamers are selected using the combinatorial chemistry method called SELEX (Systematic Evolution of Ligands by Exponential Enrichment). Compared to combinatorial libraries for phage display $\left(<10^{11}\right.$ peptides $)$ and immune repertoire of the rat $\left(<10^{8}\right.$ immunoglobulins), SELEX technology uses a larger random sequence library consisting of up to $10^{15}$ different sequences to select target-specific ap-

In vivo Tissue Engineering

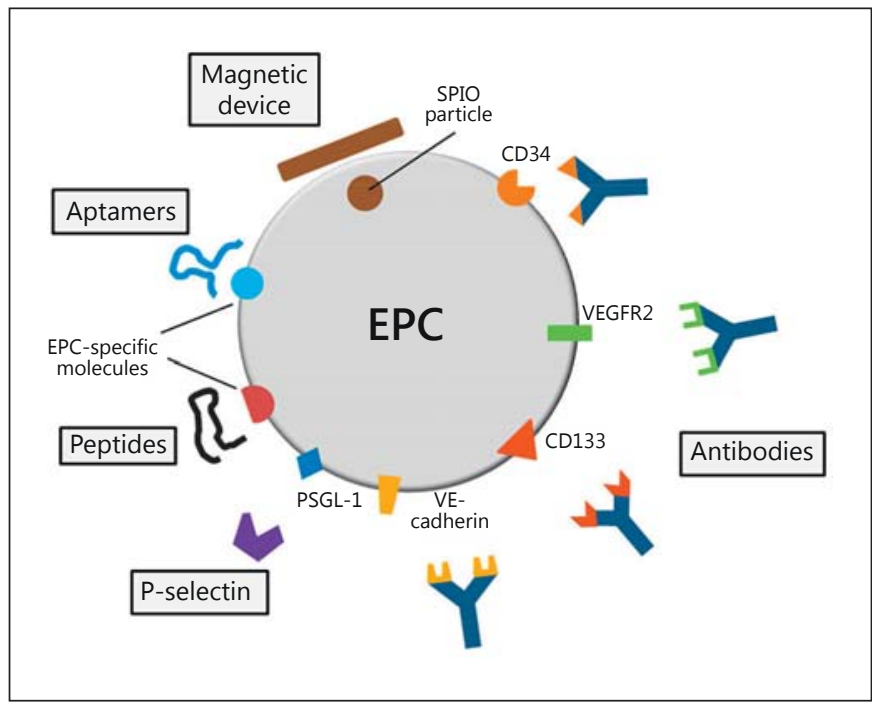

Fig. 1. Different molecules for capturing EPCs on blood-contacting materials.

tamers. Once they are selected and identified, they can be easily and chemically synthesized with high reproducibility and accuracy. They remain stable in long-term storage and undergo reversible denaturation.

Our group is currently working on aptamer-coated surfaces for small lumen vascular prostheses $(<6 \mathrm{~mm})$ to attract EPCs directly after the implantation from the bloodstream onto graft surfaces. Using SELEX technology, Hoffmann et al. [3] were able to select DNA aptamers against CD31 (PECAM-1)-positive EPCs derived from porcine peripheral blood. Promising aptamer candidates were then covalently immobilized on PTFE and polydimethylsiloxane discs by using a hemocompatible reactive 6-arm starPEG coating. StarPEG coating creates an antithrombogenic matrix on the discs and inhibits undesirable protein adsorption. In this way, the covering of immobilized capture molecules by serum proteins can be prevented and this allows a prolonged time window for the capturing of circulating EPCs onto implants. Using an in vitro closed-loop model, the ability of selected aptamers to capture EPCs from anticoagulated porcine whole blood was demonstrated under in vitro flow conditions.

\section{Antibodies}

The main EPC-specific markers on the surface of EPCs are CD34, CD133 and VEGFR2. MABs against these markers are promising as ligands for use as capture molecules. Recently, anti-VE-cadherin antibodies were also applied to attract EPCs on implant surfaces. 
The first commercially available EPC-capturing stent for in vivo endothelialization is the anti-human CD34 MAB-coated Genous ${ }^{\mathrm{TM}}$ stent (OrbusNeich Medical Technologies, Fort Lauderdale, Fla., USA) [4]. Several singlecenter [e.g. TRIAS (TRI-stent adjudication study) and HEALING-FIM (Healthy Endothelial Accelerated Lining Inhibits Neointimal Growth-First in Man)] [5, 6] and multi-center studies (e.g. HEALING II and e-HEALING) $[7,8]$ were performed using this stent to investigate its safety, feasibility and efficacy in preventing in-stent restenosis. Currently, large randomized studies are being performed to evaluate the long-term safety and efficacy in reducing neointimal hyperplasia. Despite the promising results of CD34 MAB-coated stents, the coating of ePTFE grafts with $\mathrm{CD} 34 \mathrm{MAB}$ [9] resulted in disappointing findings with regard to the neointimal hyperplasia. In pigs, rapid endothelialization of these grafts was detected. However, simultaneously, a significantly increased neointimal hyperplasia was assessed at the venous anastomosis. CD34 is not only a marker of EPCs, but is rather a pluripotent stem-cell marker, which has the ability to differentiate into smooth-muscle cells and inflammatory cells. Only $0.4 \pm 0.2 \%$ of the CD34+ cells in human peripheral blood are EPCs characterized by additional expression of CD133 and VEGFR2 [10]. Thus, 99.6\% of CD34+ cells, which are captured on these stents are not EPCs and have the capacity to differentiate into proinflammatory cells. Thereby, inversely, the intimal hyperplasia can be accelerated and lead to the occlusion of the stents. Last year, Klomp et al. [11] published their 1-year outcome of their study (high risk of restenosis TRIAS) which compared the Genous ${ }^{\mathrm{TM}}$ stent with drug-eluting stents. The inhibition of intimal hyperplasia was clearly less than with the commercially available drug-eluting stents. Thus, the Combo ${ }^{\mathrm{TM}}$ dual therapy stent [12] was developed, which has CD34 MAB on the luminal surface and a biodegradable polymer matrix containing cell-cycle-arresting sirolimus on the abluminal surface. However, by using more specific and selective capture molecules for EPCs, the prospects of success can be increased.

Markway et al. [13] oriented anti-VEGFR2 MAB using protein $\mathrm{G}$ on solid surfaces and demonstrated, under low shear stress, the ability to capture human umbilical vein endothelial cells onto a solid surface. The protein orientation improved the capturing efficiency of antibodies compared to directly adsorbed antibodies. Captured cells remained bound to the antibodies even when the shear rate was increased to the arterial shear rate. Plouffe et al. [14] also conjugated VEGFR2 MAB on the surface of a microfluidic device and demonstrated, under low shear stress, the ability of antibodies to capture in-vitro-cultivated ovine peripheral blood-derived EPCs. However, further studies with whole peripheral blood are necessary to determine the selectivity and efficiency of this antibody.

A new concept for further improving EPC-specific capture molecules was developed by Langer et al. [15] by generating bispecific molecules containing a platelet collagen receptor, glycoprotein VI, for targeting collagen and CD133 MAB. Glycoprotein VI targeted the collagen of the injured vessel wall and CD133 MAB attached to the EPCs. Previous incubation of isolated EPCs with these bispecific constructs led to enhanced reendothelialization of vascular lesions in mice compared to the treatment of EPCs with the single components of the construct alone. Thus, the use of bispecific molecules to guide EPCs to the desired regions in vivo is a promising approach. However, more selective bispecific molecules are necessary. In vivo, collagen of the injured vessel wall is recognized very quickly by platelets via collagen-binding integrin $\alpha_{2} \beta_{1}$ and glycoprotein VI. Therefore, very high amounts of constructs could be necessary to compete against platelets and to recognize the lesions with efficient numbers. Furthermore, CD133 is not only expressed on EPCs but also on hematopoietic cells, and the expression of CD133 on circulating EPCs is downregulated after mobilizing them from the bone marrow into peripheral blood [16]. Thus, EPCs which lost the CD133 expression could not be recognized by this bispecific construct.

Recently, Lim et al. [17] coated stainless steel stents with rabbit polyclonal anti-human VE-cadherin antibodies to capture EPCs. At 3 days after implantation of these stents in rabbit iliac arteries, over $90 \%$ of the surface was endothelialized. Histological analyses revealed that at 42 days, the neointimal area was significantly smaller in anti-VE-cadherin antibody-coated stents than in bare metal stents. Last year, Lee et al. [18] performed a comparison of efficacy between anti-VE-cadherin antibody-coated stents and anti-CD34 antibody-coated stents. Thereby, two different EPC capture molecules for biofunctionalization were compared, which were immobilized with the same protocol on stainless steel stents. In vitro, anti-VEcadherin antibody-coated stents captured EPCs more effectively and specifically than anti-CD34 antibody-coated stents. After stent implantation, anti-VE-cadherin antibody-coated stents were more rapidly endothelialized than anti-CD34 antibody-coated stents. At 42 days after implantation, the neointimal hyperplasia was reduced by $25 \%$ in the anti-VE-cadherin antibody-coated stents. Thus, this study demonstrated that VE-cadherin could be a more appropriate candidate for capturing EPCs. 


\section{Peptides}

Stents were coated with an integrin-binding cyclic Arg-Gly-Asp peptide (cRGD) to attract EPCs and to reduce neointimal hyperplasia. Blindt et al. [19] implanted polymer-coated cRGD-loaded stents in porcine coronary arteries. After 12 weeks, histological examinations demonstrated significantly reduced neointimal-area and percent-area stenosis compared with only polymer-coated stents or bare metal stents due to accelerated endothelialization. Recently, Joner et al. [20] demonstrated promoted endothelial cell anchorage and migration by covalent coupling of cRGD on self-expanding Nitinol stents. These studies showed that endothelialization on stents can be improved by using cRGD peptides. However, integrins with RGD-binding motifs are not only existent on EPCs, but also on other cells such as platelets. In human blood, these cells are present in high numbers and can lead to a quick coverage of cRGD-coated implants within a very short time.

Taite et al. [21] generated polyurethane vascular grafts containing the laminin-derived peptide, YIGSR (Tyr-IleGly-Ser-Arg). These grafts included further molecules in addition to the peptide, namely a diazeniumdiolate nitric oxide donor and PEG. Thus, platelet adhesion to the polyurethane surface was reduced, while endothelial cell adhesion and proliferation were enhanced. Nanofibrous matrix mimicking natural extracellular matrix can also be used for the coating of cardiovascular implants. Kushwaha et al. [22] generated nanofiber networks using two different self-assembling peptide amphiphiles, containing MMP-2 cleavage sites and YIGSR or nitric-oxide-donating residues. This matrix decreased platelet adhesion, enhanced the growth of endothelial cells and reduced the growth of smooth muscle cells. Thus, it is suitable as a coating on synthetic materials to improve the growth of captured EPCs. The use of specific capture molecules in combination with this natural extracellular matrix mimicking-coating could accelerate the in vivo self-endothelialization.

Similar to the application of SELEX technology for selection of EPC-specific aptamers, phage display technology can be used to select cell-specific peptides without prior knowledge of the composition of the cell surface molecules. Veleva et al. [23] covalently coupled a human blood outgrowth endothelial cell-binding peptide called TPS (TPSLEQRTVYAK), which was selected using phage display technology, to a methacrylic terpolymer matrix and demonstrated enhanced binding of human blood outgrowth endothelial cells in serum-free medium compared to human umbilical vein endothelial cells. The cell- specific binding was inhibited in the presence of serum proteins, however. This could be an indication for covering of capture molecules by unspecific adsorption of serum proteins. The selective binding of EPCs could be ensured by the generation of protein-resistant terpolymers.

\section{Selectins and Their Ligands}

EPCs express the P-selectin glycoprotein ligand-1 (PSGL-1) as well as the E-selectin ligand on their cell surface. These ligands can bind to E-selectin, L-selectin and $\mathrm{P}$-selectin, on inflamed and activated endothelium. However, in addition to the selectin-binding ligands, EPCs also exhibit both E-selectin and L-selectin on their surface. Sialyl Lewis ${ }_{\mathrm{X}}\left(\mathrm{sLe}_{\mathrm{X}}\right)$ is an oligosaccharide with selectin-binding ability. Biofunctionalization of collagen matrices with $\mathrm{sLe}_{\mathrm{x}}$ led to enhanced adhesion of EPCs on the matrices [24] and increased the neovascularization of collagen matrices after the implantation in ischemic hindlimbs. Narasipura et al. [25] generated P-selectincoated nanotubes and demonstrated the ability to capture CD34+ cells from human bone marrow-derived mononuclear cells. Implanted P-selectin-coated plastic tubes in the femoral arteries of rats demonstrated increased capture of CD34+ cells from circulating blood when compared to noncoated control tubes [26]. However, the fact that only $28 \%$ of the captured cells on the P-selectin-coated tubes were CD34+ cells, confirms that PSGL-1 is not only expressed on EPCs, but also on all white blood cells. Therefore, P-selectin is not selective only for circulating EPCs in the peripheral blood. Unfortunately, this also applies to $\mathrm{sLe}_{\mathrm{x}}$ as it is expressed on monocytes and granulocytes.

\section{Magnetic Molecules}

EPCs can be isolated and labeled with superparamagnetic iron oxide (SPIO) particles. After injection into the patient's body, a magnetic device can be applied to attract EPCs to the desired location. To this end, Pislaru et al. [27] generated magnetizable stents by coating stainlesssteel stents with nickel. Magnetized stents were implanted in porcine coronary and femoral arteries and captured 6- to 30-fold more SPIO microsphere-loaded EPCs than nonmagnetized stents. In further studies, Kyrtatos et al. [28] demonstrated enhanced localization of SPIO nanoparticle-loaded EPCs at the site of injured rat carotid arteries by using an external magnetic device. Despite promising results, this technique needs the isolation and cultivation of EPCs in vitro and the subsequent labeling of them with SPIO particles under GMP conditions. So it is time-consuming and expensive and can lead to a bacte- 
rial contamination or to a cell alteration. After SPIO labeling of EPCs, Yang et al. [29] demonstrated an impairment of EPC migration ability; however, proliferation and apoptosis were not affected. Additional studies investigating the influence of released SPIO particles on the surrounding tissue are also needed. The induction of inflammation can have an adverse effect on graft patency by triggering the intimal hyperplasia or causing the occlusion of implanted grafts.

\section{Further Investigations to Improve Self-Endothelialization of Blood-Contacting Materials}

Being able to generate hemocompatible surface coatings on the surface of blood-contacting materials is an important factor for the successful and selective capturing of EPCs from the bloodstream. The prevention of the unspecific adsorption of serum proteins and adhesion of thrombocytes ensures the accessibility of the capture molecules for EPCs. Furthermore, the preservation of the functionality of homing-factor-mimetic molecules after their conjugation onto hemocompatible surfaces is a further requirement for a successful recognition of EPCs in vivo. A site-specific conjugation of capture molecules and probably the use of spacers between the hemocompatible coating and capture molecules, which enable the capture molecules to protrude from the surface into the bloodstream could improve the speed and colonization of blood-contacting surfaces with EPCs. Further investigations can be performed concerning the necessity of growth factor immobilization in the hemocompatible coating to support the differentiation of EPCs into endothelial cells. Most importantly, however, is the use of highly selective EPC capture molecules. Therefore, the identification of new EPC markers which are present only on these stem cells is crucial. For this purpose, comparative protein analyses or microarray analyses can be performed to identify new markers on EPCs. In this regard, cell SELEX and phage display are very promising technologies for obtaining new EPC-binding ligands. Both techniques can be performed without knowing the composition of the cell surface molecules. By incubation with negative cells, e.g. with mononuclear blood cells, and elimination of unspecific binding molecules, the binding of aptamers or peptides to only the desired cells can be ensured. After the selection process, target identification can be performed and new previous unknown markers on EPCs can be identified.

\section{Conclusion}

The functionalization of blood-contacting artificial surfaces with EPC homing-factor-mimetic molecules is an auspicious strategy towards the biologization of medical products. Using these ready-to-use medical products, the in vivo endothelialization of artificial surfaces can take place with patient's autologous stem cells in his/her own body. However, the success of this approach depends heavily on the use of highly selective capture molecules for in vivo applications. Thus, the search continues to find the most suitable molecules. This can be achieved by using new methods for target identification, better characterization of EPC surface molecules, their homing behavior and involved interactions. The immobilization of capture molecules on substrates, which support the functionality and accessibility, will further improve the selfendothelialization of blood-contacting surfaces. Thus, this approach can be used for biofunctionalization of several blood-contacting implants and devices to obtain the best hecompatibility for long-term use and to prevent the recognition of them as foreign bodies.

\section{References}

1 Wendel HP, Ziemer G: Coating techniques to improve the hemocompatibility of artificial devices used for extracorporeal circulation. Eur J Cardiothorac Surg 1999;16:342-350.

-2 Li S, Henry JJ: Nonthrombogenic approaches to cardiovascular bioengineering. Annu Rev Biomed Eng 2011;13:451-475.

- 3 Hoffmann J, et al: Immobilized DNA aptamers used as potent attractors for porcine endothelial precursor cells. J Biomed Mater Res A 2008;84:614-621.
4 Klomp M, Beijk MA, de Winter RJ: Genous endothelial progenitor cell-capturing stent system: a novel stent technology. Expert Rev Med Devices 2009;6:365-375.

5 Aoki J, Serruys PW, van Beusekom H, Ong AT, McFadden EP, Sianos G, van der Giessen WJ, Regar E, de Feyter PJ, Davis HR, Rowland S, Kutryk MJ: Endothelial progenitor cell capture by stents coated with antibody against CD34: the HEALING-FIM (Healthy Endothelial Accelerated Lining Inhibits Neointi- mal Growth-First In Man) Registry. J Am Coll Cardiol 2005;45:1574-1579.

-6 Beijk MA, Klomp M, van GN, Koch KT, Henriques JP, Baan J, Vis MM, Tijssen JG, Piek JJ, de Winter RJ: Two-year follow-up of the Genous endothelial progenitor cell capturing stent versus the Taxus Liberte stent in patients with de novo coronary artery lesions with a high risk of restenosis: a randomized, singlecenter, pilot study. Catheter Cardiovasc Interv 2011;78:189-195.
Avci-Adali/Stoll/Wilhelm/Perle/ Schlensak/Wendel 
7 Duckers HJ, et al: Accelerated vascular repair following percutaneous coronary intervention by capture of endothelial progenitor cells promotes regression of neointimal growth at long-term follow-up: final results of the HEALING II trial using an endothelial progenitor cell capturing stent (Genous R stent). Euro Intervention 2007;3:350-358.

$>8$ Silber S, et al: Clinical results after coronary stenting with the Genous Bio-engineered $\mathrm{R}$ stent: 12-month outcomes of the e-HEALING (Healthy Endothelial Accelerated Lining Inhibits Neointimal Growth) worldwide registry. EuroIntervention 2011;6:819-825.

-9 Rotmans JI, Heyligers JM, Verhagen HJ, Velema E, Nagtegaal MM, de Kleijn DP, de Groot FG, Stroes ES, Pasterkamp G: In vivo cell seeding with anti-CD34 antibodies successfully accelerates endothelialization but stimulates intimal hyperplasia in porcine arteriovenous expanded polytetrafluoroethylene grafts. Circulation 2005;112:12-18.

10 Peichev M, et al: Expression of VEGFR-2 and AC133 by circulating human CD34(+) cells identifies a population of functional endothelial precursors. Blood 2000;95:952-958.

$>11$ Klomp M, et al: 1-year outcome of TRIAS HR (TRI-stent adjudication study-high risk of restenosis) a multicenter, randomized trial comparing Genous endothelial progenitor cell capturing stents with drug-eluting stents. JACC Cardiovasc Interv 2011;4:896-904.

12 Granada JF, et al: Development of a novel prohealing stent designed to deliver sirolimus from a biodegradable abluminal matrix. Circ Cardiovasc Interv 2003;3:257-266.

-13 Markway BD, McCarty OJ, Marzec UM, Courtman DW, Hanson SR, Hinds MT: Capture of flowing endothelial cells using surfaceimmobilized anti-kinase insert domain receptor antibody. Tissue Eng Part C Methods 2008;14:97-105.

$>14$ Plouffe BD, Kniazeva T, Mayer JE Jr, Murthy SK, Sales VL: Development of microfluidics as endothelial progenitor cell capture technology for cardiovascular tissue engineering and diagnostic medicine. FASEB J 2009;23: 3309-3314.
15 Langer HF, et al: Capture of endothelial progenitor cells by a bispecific protein/monoclonal antibody molecule induces reendothelialization of vascular lesions. J Mol Med 2010;88:687-699.

16 Hristov M, Erl W, Weber PC: Endothelial progenitor cells: mobilization, differentiation, and homing. Arterioscler Thromb Vasc Biol 2003;23:1185-1189.

17 Lim WH, et al: Stent coated with antibody against vascular endothelial-cadherin captures endothelial progenitor cells, accelerates re-endothelialization, and reduces neointimal formation. Arterioscler Thromb Vasc Biol 2011;31:2798-2805.

18 Lee JM, et al: Comparison of endothelialization and neointimal formation with stents coated with antibodies against CD34 and vascular endothelial-cadherin. Biomaterials 2012;33:8917-8927.

19 Blindt R, et al: A novel drug-eluting stent coated with an integrin-binding cyclic ArgGly-Asp peptide inhibits neointimal hyperplasia by recruiting endothelial progenitor cells. J Am Coll Cardiol 2006;47:1786-1795.

20 Joner M, Cheng Q, Schonhofer-Merl S, Lopez M, Neubauer S, Mas-Moruno C, Laufer B, Kolodgie FD, Kessler H, Virmani R: Polymerfree immobilization of a cyclic RGD peptide on a Nitinol stent promotes integrin-dependent endothelial coverage of strut surfaces. J Biomed Mater Res B Appl Biomater 2012;100: 637-645.

21 Taite LJ, Yang P, Jun HW, West JL: Nitric oxide-releasing polyurethane-PEG copolymer containing the YIGSR peptide promotes endothelialization with decreased platelet adhesion. J Biomed Mater Res B Appl Biomater 2008;84:108-116.
22 Kushwaha M, Anderson JM, Bosworth CA, Andukuri A, Minor WP, Lancaster JR Jr, Anderson PG, Brott BC, Jun HW: A nitric oxide releasing, self-assembled peptide amphiphile matrix that mimics native endothelium for coating implantable cardiovascular devices. Biomaterials 2010;31;1502-1508.

23 Veleva AN, Heath DE, Cooper SL, Patterson C: Selective endothelial cell attachment to peptide-modified terpolymers. Biomaterials 2008;29:3656-3661.

24 Suuronen EJ, Zhang P, Kuraitis D, Cao X, Melhuish A, McKee D, Li F, Mesana TG, Veinot JP, Ruel M: An acellular matrix-bound ligand enhances the mobilization, recruitment and therapeutic effects of circulating progenitor cells in a hindlimb ischemia model. FASEB J 2009;23:1447-1458.

25 Narasipura SD, Wojciechowski JC, Charles N, Liesveld JL, King MR: P-selectin-coated microtube for enrichment of CD34+ hematopoietic stem and progenitor cells from human bone marrow. Clin Chem 2008;54:77-85.

26 Wojciechowski JC, Narasipura SD, Charles N, Mickelsen D, Rana K, Blair ML, King MR: Capture and enrichment of CD34-positive haematopoietic stem and progenitor cells from blood circulation using P-selectin in an implantable device. Br J Haematol 2008;140: 673-681.

27 Pislaru SV, Harbuzariu A, Gulati R, Witt T, Sandhu NP, Simari RD, Sandhu GS: Magnetically targeted endothelial cell localization in stented vessels. J Am Coll Cardiol 2006;48: 1839-1845.

28 Kyrtatos PG, Lehtolainen P, JunemannRamirez M, Garcia-Prieto A, Price AN, Martin JF, Gadian DG, Pankhurst QA, Lythgoe MF: Magnetic tagging increases delivery of circulating progenitors in vascular injury. JACC. Cardiovasc Interv 2009;2:794-802.

29 Yang JX, Tang WL, Wang XX: Superparamagnetic iron oxide nanoparticles may affect endothelial progenitor cell migration ability and adhesion capacity. Cytotherapy 2010;12: 251-259.

In vivo Tissue Engineering 